\title{
AOTrauma-Reisestipendium zum Hospital for Special Surgery (HSS) in NYC
}

Andreas Baranowski

\section{"If you reject the food, ignore the customs, fear the religion and avoid the people, you might better stay at home." - James Michener}

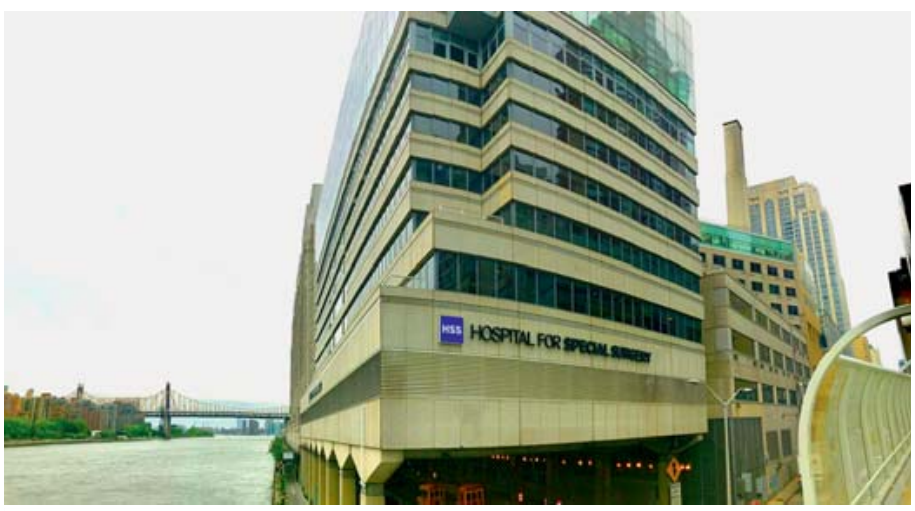

Abb. 1 Das HSS in NY.

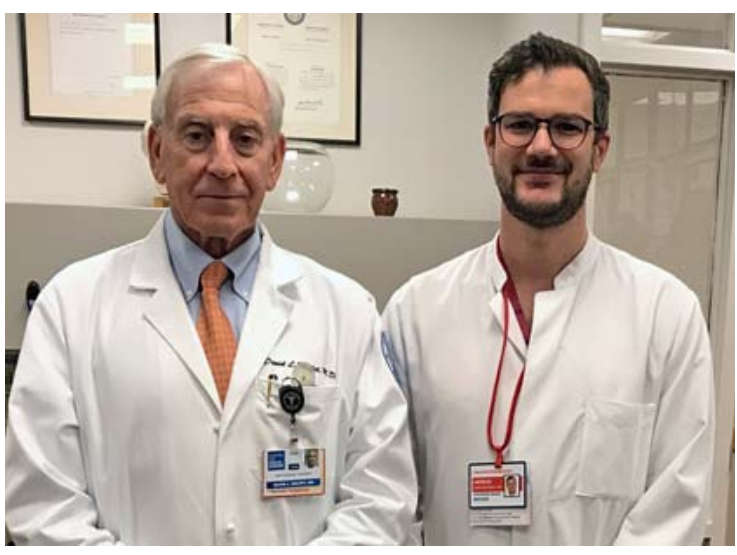

- Abb. 2 David Helfet und Andreas Baranowski.

Die anschließenden Operationen, die sich oft bis in die späten Abendstunden hinzogen, wurden teilweise in Operationssälen des New York-Presbyterian Hospitals durchgeführt.

Neben den Operationen, bei denen ich Dr. Wellman begleitete, hatte ich auch die Gelegenheit, von David L. Helfet, Joseph M. Lane und William Ricci zu lernen und Fragen zu stellen. Über eine Kamera im Lampengriff wurde das OP-Feld auf Flachbildschirme übertragen, sodass man stets einen hervorragenden Überblick hatte. Das Team nahm mich sehr freundlich auf und zeigte sich interessiert an den Gemeinsamkeiten und Unterschieden zur operativen Versorgung am Zentrum für Orthopädie und Unfallchirurgie der Uniklinik Mainz.

Spannend und neu für mich waren insbesondere das Erlernen der Technik zur Verwendung des Fibula-Allografts bei der Versorgung der proximalen Humerusfraktur sowie Einblicke in die vielseitige Einsetzbarkeit der meshPlatte oder des autologen bone marrow autografts (BMAG) in der Technik nach Hernigou. 
Auch außerhalb des OPs gab es viel zu lernen. An 2 Tagen der Woche fand die Sprechstunde statt, in der Dr. Wellman bis zu 45 Patienten pro Tag zusammen mit seinem PA (Physician Assistant) Ethan C. Jensen behandelte. Der PA besitzt in New York weitreichende Befugnisse, darf beispielsweile Rezepte ausstellen, Krankengymnastik verordnen, die Patienten untersuchen und die Briefe der Sprechstunde verfassen. Dadurch stellt er eine wesentliche Entlastung für den Arzt dar. Die gemeinsame Arbeit mit David Wellman und Ethan Jensen machte viel Freude und zeigte mir die Vorteile des „physician assistant“-Systems, welches in dieser Form in Deutschland noch nicht etabliert ist.

Das Reisestipendium der AOTrauma Deutschland verschaffte mir die einmalige Gelegenheit, die Arbeit international herausragender Unfallchirurgen für 4 Wochen begleiten zu dürfen und im kollegialen und freundschaftlichen Gespräch besondere Einblicke zu gewinnen. Dabei waren nicht nur die Unterschiede in der Patientenversorgung interessant, sondern vor allem auch die Gemeinsamkeiten bei den universell zur Anwendung kommenden Osteosynthesetechniken der AO. Für diese Möglichkeit der fachlichen Bereicherung möchte ich mich bei der AOTrauma Deutschland herzlich bedanken. Ich kann allen Assistenz- und Fachärzten wärmstens empfehlen, sich für die Reisestipendien der $\mathrm{AO}$ zu bewerben und im internationalen Austausch den eigenen Horizont zu erweitern.

Andreas Baranowski, Universitätsklinikum Mainz E-Mail: andreas.baranowski@unimedizin-mainz.de

Bibliografie

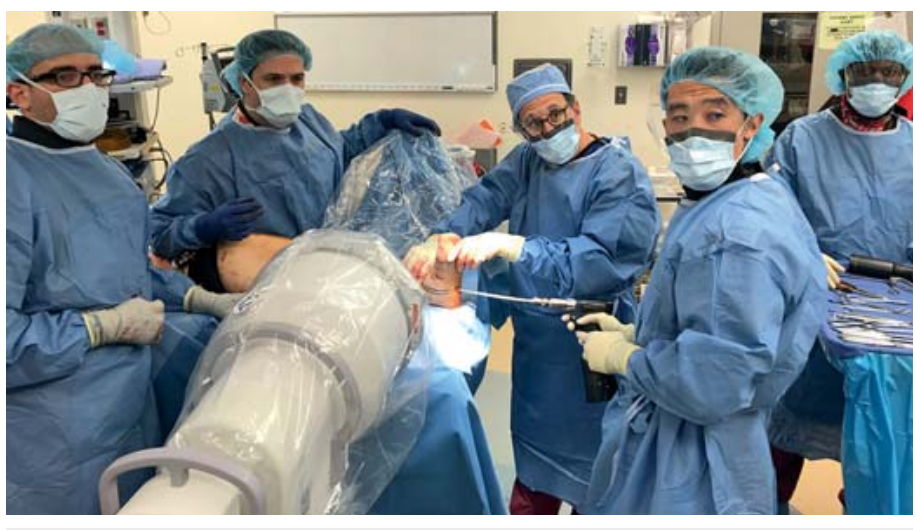

- Abb. 3 Im OP.

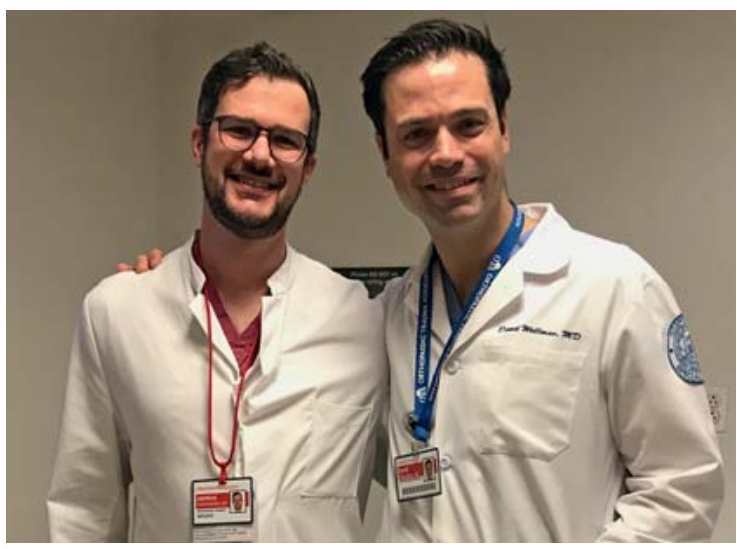

- Abb. 4 Mit Gastgeber David Wellman.

DOI https://doi.org/10.1055/a-0647-2582

OP-JOURNAL 2018; 34: 184-185 @ Georg Thieme Verlag KG Stuttgart · New York ISSN 0178-1715 